\section{Exame ocupacional periódico: oportunidade de diagnóstico e monitoramento de doença crônica não transmissível em homens}

\author{
Periodic occupational examination: an opportunity \\ for diagnosis and monitoring of chronic \\ noncommunicable diseases in men
}

\section{Examen ocupacional periódico: oportunidad de diagnóstico y supervisión de enfermedad crónica no transmisible en hombres}

Tamyres Araújo Andrade Donato 1

Roberta Mendes Abreu Silva 1

Amanda Cristina de Souza Andrade 2

Clávdia Nicolaevna Kochergin 1

Danielle Souto de Medeiros 1

Daniela Arruda Soares 1

José Andrade Louzado 1

Kelle Oliveira Silva 1

Matheus Lopes Cortes 1

Sóstenes Mistro 1

Welma Wildes Cunha Coelho Amorim 3

Márcio Galvão Guimarães de Oliveira 1

Vanessa Moraes Bezerra 1

\section{Resumo}

Objetivou-se estimar a prevalência de hipertensão arterial, como principal marcador de doença crônica não transmissível (DCNT), e identificar os fatores modificáveis associados, em trabalhadores homens. Foram utilizados dados da linha de base de um estudo longitudinal com uma amostra de 1.024 trabalhadores homens com 18 anos ou mais de um município do Nordeste do Brasil. O marcador de DCNT foi a hipertensão arterial, definida por pressão arterial sistólica $\geq 140 \mathrm{mmHg}$ e/ou pressão arterial diastólica $\geq 90 \mathrm{mmHg}$ e/ou diagnóstico prévio de hipertensão arterial e/ou uso de medicamentos anti-hipertensivos. Empregou-se a regressão de Poisson com variância robusta, adotando a entrada hierárquica de variáveis. Foram calculadas frações atribuíveis populacionais (FAP) para as variáveis de estilo de vida, a fim de dimensionar o impacto dos fatores modificáveis na saúde dos trabalhadores. A prevalência da hipertensão arterial nesta população foi de 28,6\% (IC95\%: 25,9-31,5), os fatores distais: idade > 40 anos, cor da pele preta e renda familiar $\geq 3$ salários mínimos; fatores intermediários: consumo abusivo de álcool, consumo de tabaco, percepção de um consumo elevado de sal e inatividade física e o fator proximal: sobrepeso e obesidade associaram-se positivamente com a hipertensão arterial. O cálculo da FAP permitiu observar que se ocorresse a redução ou eliminação de hábitos e comportamentos relacionados ao estilo de vida deste público, reduziria em 56,1\% a prevalência da DCNT estudada. A identificação de fatores modificáveis e como estes podem interferir negativamente na saúde de trabalhadores homens possibilita o planejamento de intervenções no próprio local de trabalho, a fim de alcançar o maior número de indivíduos, visando reduzir os efeitos deletérios das DCNT.

Exames Médicos; Diagnóstico; Doenças Não Transmissíveis; Saúde do Trabalhador; Homens

\author{
Correspondência \\ T. A. A. Donato \\ Instituto Multidisciplinar em Saúde, Universidade Federal da \\ Bahia. \\ Rua Hormindo Barros 58, Quadra 17, Lote 58, Vitória da \\ Conquista, BA 45029-094, Brasil. \\ tamyres_donato@hotmail.com \\ ${ }^{1}$ Instituto Multidisciplinar em Saúde, Universidade Federal da \\ Bahia, Vitória da Conquista, Brasil. \\ 2 Universidade Federal de Minas Gerais, Belo Horizonte, Brasil. \\ 3 Universidade Estadual do Sudoeste da Bahia, Vitória da \\ Conquista, Brasil.
}




\section{Introdução}

O local de trabalho é caracterizado como um ambiente que exerce influência sobre a saúde do trabalhador, visto que eles permanecem a maioria do tempo no ambiente ocupacional, podendo desenvolver hábitos de vida não saudáveis 1 e adoecimento por agravos crônicos, inicialmente assintomáticos 2 . A triagem da situação de saúde dos trabalhadores no ambiente laboral oportuniza o diagnóstico de doenças crônicas de maior prevalência, como a hipertensão arterial 2.

A hipertensão arterial, uma das principais doenças crônicas não transmissíveis (DCNT), configura-se como um fator de risco para doenças cardiovasculares (DCV) e afeta um bilhão de pessoas em todo o mundo, com impacto elevado na perda da produtividade do trabalho e renda familiar 3,4, afetando em maior número os homens 2 .

O trabalho lidera a lista das preocupações masculinas, direcionando sua atenção para o sustento da família, mais do que para os cuidados com a própria saúde, reduzindo a procura aos serviços de saúde 5,6. Isso pode acarretar afastamentos precoces do trabalho 2 e diagnósticos tardios de doenças que poderiam ser controladas ou tratadas, levando a complicações muitas vezes irreversíveis ${ }^{5}$, podendo refletir na economia do país 3 .

Os trabalhadores homens têm se destacado por apresentar altas prevalências de condições crônicas desfavoráveis, desconhecimento das mesmas e consequente menor oportunidade de tratamento e controle 5 . O contexto de vida do trabalhador, predominantemente com rotinas extensas, cargas horárias laborais exaustivas e alta pressão por produtividade podem contribuir no surgimento de hábitos e comportamentos inadequados, sendo estes indicados como principais marcadores no surgimento de problemas de saúde 7,8,9. A realização de rastreio para DCNT no ambiente trabalho, além de contribuir com a prevenção, se apresenta como uma oportunidade de identificar doenças de alta relevância para saúde pública e direcionar estratégias de monitoramento, que pode ocorrer de maneira mais precoce e assim evitar consequências desfavoráveis 1,2 .

A Organização Mundial da Saúde (OMS) definiu um conjunto de metas globais para redução das DCNT 10. Para o alcance dessas metas, as empresas podem usar de recursos disponíveis para orientação, educação e motivação a fim de incentivar mudanças de estilo de vida 11,12. Essas medidas envolvem desde o contato individual até a utilização de fontes de informações coletivas, como reuniões, palestras e vídeos.

O presente estudo teve como objetivo estimar a prevalência de hipertensão arterial, como principal marcador de DCNT e identificar os fatores modificáveis associados, em trabalhadores homens, a fim de sinalizar os grupos prioritários para rastreio, estimando o efeito das mudanças de estilo de vida sobre o evento estudado. Toda essa análise permitirá posteriormente definir estratégias de enfrentamento, prevenção e promoção da saúde dos trabalhadores.

\section{Métodos}

Trata-se de um estudo transversal, que utilizou dados do projeto longitudinal HealthRise Vitória da Conquista 13. Vitória da Conquista é o terceiro maior município do Estado da Bahia, localizado na Região Nordeste do Brasil 14. A pesquisa foi realizada com uma população masculina, de trabalhadores atendidos na unidade do Serviço Social da Indústria (SESI), em Vitória da Conquista. O SESI é uma entidade privada, sem fins lucrativos, cuja missão é qualificar a força de trabalho e promover a saúde do trabalhador. Atende diferentes ocupações, a exemplo de trabalhadores das indústrias, construção civil, trabalhadores administrativos, saúde e educação 15.

Os dados do presente trabalho são provenientes da linha de base realizada entre agosto de 2017 a julho de 2018.

Esta pesquisa foi aprovada pelo Comitê de Ética em Pesquisa do Instituto Multidisciplinar em Saúde da Universidade Federal da Bahia (CAEE 62259116.0.0000.5556) e os participantes que aceitaram participar deram seu consentimento informado por escrito.

Foram considerados para o cálculo amostral todos os trabalhadores com 18 anos ou mais, residentes no município, que compareceriam à unidade para as consultas periódicas com o médico do trabalho, totalizando 2.014 trabalhadores. Utilizou-se nível de 95\% de confiança, prevalência de 
hipertensão arterial em homens adultos brasileiros de 25,8\% 11 e um erro tolerável de $2 \%$. A amostra final foi de 1.047 trabalhadores considerando-se $10 \%$ de perdas.

\section{Coleta dos dados}

Foram realizadas entrevistas individuais, por entrevistadores devidamente capacitados, utilizando tablets contendo o software KoboToolbox (https://www.kobotoolbox.org/). O local de coleta de dados ocorreu em salas reservadas, no SESI, e os trabalhadores que iam para consultas periódicas com o médico do trabalho eram convidados a participarem da pesquisa. Os dados coletados foram sincronizados de forma criptografada. $\mathrm{O}$ instrumento de entrevistas utilizado foi um questionário semiestruturado baseado no questionário da Pesquisa Nacional de Saúde 16, no Questionário Internacional de Atividade Física (IPAQ) 17, em sua versão curta, e na escala de qualidade de vida EUROHIS (índice europeu de qualidade de vida) 18 .

Foram realizadas três medidas de pressão arterial (com intervalo de um minuto entre cada aferição) utilizando-se o esfigmomanômetro digital da marca Omron modelo HEM-7113 (Omron Healthcare Brasil, São Paulo, Brasil), validado internacionalmente 19. As aferições foram realizadas após as entrevistas, visando garantir que os indivíduos estivessem em repouso, sentados, com as pernas descruzadas, os pés apoiados no chão, o dorso recostado na cadeira e relaxado, com o braço esquerdo apoiado sobre a mesa à altura do coração. Certificou-se de que os mesmos: não estivessem com a bexiga cheia, não tivessem praticado exercícios físicos há pelo menos 60 minutos, não tivessem ingerido bebidas alcoólicas, café ou alimento e não tivessem fumado nos últimos 30 minutos. $\mathrm{O}$ valor final da pressão arterial foi obtido pela média das duas últimas medidas 20 . As aferições realizadas no âmbito do estudo eram conferidas com as aferições realizadas pelo médico do trabalho, a fim de garantir a fidedignidade dos dados.

O peso foi verificado com os indivíduos descalços, vestindo roupas leves, sem portar acessórios e objetos em bolsos, em balança eletrônica portátil digital, da marca Seca 813 (Seca GmbH \& Co. KG, Hamburgo, Alemanha), com capacidade para $200 \mathrm{~kg}$ e precisão de $100 \mathrm{~g}$. Para a aferição da altura foi utilizado o estadiômetro portátil da marca NutriVida (NutriVida Home Care, Natal, Brasil), composto por extensor com escala numérica e base para apoio dos pés, com os indivíduos descalços e em posição ereta. A circunferência da cintura foi medida utilizando-se uma fita métrica, sendo considerado o ponto médio entre a borda inferior da última costela e a borda superior da crista ilíaca.

\section{Variáveis estudadas}

A variável dependente deste estudo foi a hipertensão arterial, escolhida por ser umas das DCNT mais prevalentes em diferentes populações 2,6,8. Foram classificados hipertensos todos aqueles com diagnóstico médico prévio de hipertensão arterial e/ou que tiveram valores de pressão arterial sistólica $\geq 140 \mathrm{mmHg}$ e/ou pressão arterial diastólica $\geq 90 \mathrm{mmHg}$ e/ou faziam uso de medicamentos anti-hipertensivos 20 .

As variáveis independentes foram estruturadas nos seguintes blocos: sociodemográficas, estilo de vida e índices antropométricos.

O primeiro bloco foi composto por variáveis sociodemográficas que são comumente associadas a hipertensão arterial $21 \mathrm{e}$, portanto, importante de serem consideradas como fatores de ajustes em análises. Foram inseridas: idade em anos (18-29 anos, 30-39 anos, 40 anos ou mais 11), raça (branca, preta, amarela, parda e indígena), estado civil (solteiro, casado ou com companheira(o), separado/viúvo), renda familiar (menor do que 2 salários mínimos, de 2 salários até menos de 3 salários mínimos ou 3 salários ou mais 21,22) e anos de estudo (0-8 anos, 9-11 anos, 12 anos ou mais 22).

Quanto ao trabalho, os turnos foram dicotomizados em diurno/noturno e apenas diurno. Os trabalhadores da presente pesquisa eram provenientes de diferentes empresas do município, representando ramos de ocupação variados. O cargo exercido foi classificado de acordo com a Classificação Brasileira de Ocupações 23, sendo dicotomizado em braçal (trabalhadores das indústrias, construção civil, serviços gerais e serviços braçais avulsos) e não braçal (trabalhadores administrativos, saúde e educação) 24 . 
O segundo bloco de variáveis foi composto por aquelas consideradas como foco de intervenção por serem passíveis de modificação. Foi avaliado o consumo abusivo de álcool, identificado por aqueles que relataram ingerir cinco ou mais doses de bebida alcoólica, em uma única ocasião, nos últimos 30 dias 25 . Uma dose padrão contém, aproximadamente, $10 \mathrm{~g}$ de álcool puro 25 . Aqueles que relataram fumar no período da pesquisa foram classificados positivamente para o consumo de tabaco 26 .

Quanto aos hábitos alimentares, foi considerada a frequência semanal do consumo de frutas, saladas e legumes crus, cada variável categorizada em $\geq 5$ dias por semana (adequado) e $<5$ dias por semana (inadequado) 27 . O consumo de alimentos ultraprocessados foi classificado como positivo por quem referiu substituir as refeições por esses alimentos em pelo menos um dia da semana ${ }^{27}$. Para determinar a percepção do consumo de sal, foi utilizada a seguinte pergunta: "Considerando a comida preparada na hora e os alimentos industrializados, o(a) Sr(a) acha que seu consumo de sal é?”, sendo recategorizada em "baixo/muito baixo; adequado; alto/muito alto" 28.

A atividade física foi avaliada pelo IPAQ curto ${ }^{17}$, sendo mensurada através da multiplicação da frequência semanal (dias) pela duração média (minutos) da prática de atividade física moderada e vigorosa. O tempo dispendido em atividades vigorosas foi multiplicado por dois. Apenas atividades desempenhadas por pelo menos 10 minutos contínuos foram validadas. Foram considerados inativos aqueles que praticavam menos do que 150 minutos de atividade física por semana 29,30.

A qualidade de vida foi avaliada pelo EUROHIS-QL-8 18. A escala é composta por oito itens que abrangem a saúde em geral, as relações interpessoais, financeiras e domiciliares. A qualidade de vida é calculada somando os oito itens, e as pontuações mais altas indicam melhor qualidade de vida 18.

De acordo com as medidas objetivas realizadas, foram descritos o índice de massa corporal dos participantes 31 , sendo considerado: $<18,5 \mathrm{~kg} / \mathrm{m}^{2}$ "magro ou baixo peso", entre $18,5-24,9 \mathrm{~kg} / \mathrm{m}^{2}$ "normal", entre $25-29,9 \mathrm{~kg} / \mathrm{m}^{2}$ "sobrepeso" e IMC $>30 \mathrm{~kg} / \mathrm{m}^{2}$ "obesidade". A circunferência da cintura foi categorizada em adequada $(<94 \mathrm{~cm})$, aumentada $(\geq 94 \mathrm{~cm})$ e muito aumentada $(\geq 102 \mathrm{~cm}) 32$.

\section{Análise estatística}

Foi feita uma estimativa da prevalência da hipertensão arterial entre os trabalhadores homens e intervalo de 95\% de confiança (IC95\%). Para verificar os fatores associados à hipertensão arterial, foram feitas análises bivariada e multivariada com estimativas de razões de prevalência (RP) e cálculo dos respectivos valores de p e IC95\% por meio de regressão de Poisson com variância robusta.

Para a seleção das variáveis destinadas à modelagem, foram usadas aquelas que apresentaram significância estatística de até $20 \%(\mathrm{p}<0,20)$ na análise bivariada. Foi realizada a entrada hierárquica das variáveis em blocos 32,33, obedecendo a seguinte ordem, conforme estudos prévios 32,34: sociodemográficas, estilo de vida e índices antropométricos. Consideramos associação estatisticamente significativa quando o valor de $\mathrm{p} \leq 0,05$, após ajuste para os fatores do mesmo bloco e dos blocos hierarquicamente superiores. Foi utilizado para comparação entre os modelos o critério de Akaike (AIC) 35.

A fração atribuível populacional (FAP) foi calculada para alguns fatores modificáveis. Ela estima a proporção da doença ou evento relacionado à saúde que seria prevenido na população caso o fator de risco fosse eliminado. Quando as estimativas de o risco relativo (RR) ajustado são utilizadas recomenda-se a utilização da equação: $\mathrm{FAP}=\operatorname{Pc}($ RRajustada -1$) /($ RRajustada $)$ em que Pc é a proporção de exposição entre os casos da doença, RRajustada é o risco relativo ajustado por variáveis de confusão. Em estudos transversais a FAP pode ser calculada como uma função da razão de prevalências 36 . O programa Stata versão 14 (https://www.stata.com) foi utilizado na análise dos dados.

\section{Resultados}

Do total de 1.047 trabalhadores previstos na amostra, 1.024 participaram deste estudo, sendo observada uma perda de $2,1 \%$ devido a recusas.

Foi observado um maior percentual de participantes entre 30-39 anos (40,3\%). A maioria dos trabalhadores se autorreferiram pardos (54,7\%), casados (65,1\%), com escolaridade entre 9-11 anos de estudo (52,9\%), exerciam trabalho braçal (62\%) e trabalhavam em turnos diurnos $(79,2 \%)$. Quanto à renda familiar, 36,7\% relataram receber menos do que 2 salários mínimos (Tabela 1). 
Tabela 1

Características da população de trabalhadores homens de um município do Nordeste do Brasil, 2017-2018.

\begin{tabular}{|c|c|c|c|}
\hline Variável & $n *$ & $\%$ & IC95\% \\
\hline \multicolumn{4}{|l|}{ Sociodemográficas } \\
\hline \multicolumn{4}{|l|}{ Idade (anos) } \\
\hline $18-29$ & 329 & 32,1 & $29,3-35,1$ \\
\hline $30-39$ & 413 & 40,3 & $37,4-43,4$ \\
\hline 40 ou mais & 282 & 27,5 & $24,9-30,4$ \\
\hline \multicolumn{4}{|l|}{ Raça } \\
\hline Branca & 197 & 19,4 & $17,1-22,0$ \\
\hline Preta & 217 & 21,4 & $19,0-24,0$ \\
\hline Amarela & 28 & 2,8 & $1,9-3,9$ \\
\hline Parda & 555 & 54,7 & $51,7-57,8$ \\
\hline Indígena & 17 & 1,7 & $1,0-2,7$ \\
\hline \multicolumn{4}{|l|}{ Estado civil } \\
\hline Solteiro & 291 & 28,4 & $25,7-31,3$ \\
\hline Casado ou com companheiro(a) & 667 & 65,1 & $62,2-68,0$ \\
\hline Separado/Viúvo & 66 & 6,5 & $5,1-8,1$ \\
\hline \multicolumn{4}{|l|}{ Renda familiar (salários mínimos) ** } \\
\hline$<2$ & 367 & 36,7 & $33,8-39,8$ \\
\hline $2<3$ & 280 & 28,0 & $25,3-30,9$ \\
\hline$\geq 3$ & 352 & 35,2 & $32,3-38,3$ \\
\hline \multicolumn{4}{|l|}{ Anos de estudo (anos) } \\
\hline $0-8$ & 287 & 28,1 & $25,4-30,9$ \\
\hline $9-11$ & 541 & 52,9 & $49,9-56,0$ \\
\hline$\geq 12$ & 194 & 19,0 & $16,7-21,5$ \\
\hline \multicolumn{4}{|l|}{ Turno de trabalho } \\
\hline Diurno/Noturno & 210 & 20,8 & $18,4-23,4$ \\
\hline Diurno & 799 & 79,2 & $76,6-51,6$ \\
\hline \multicolumn{4}{|l|}{ Cargos de trabalho } \\
\hline Não braçal & 389 & 38,0 & $35,1-41,0$ \\
\hline Braçal & 635 & 62,0 & $59,0-64,9$ \\
\hline \multicolumn{4}{|l|}{ Estilo de vida } \\
\hline \multicolumn{4}{|l|}{ Consumo abusivo de álcool } \\
\hline Não & 693 & 67,9 & $64,9-7,7$ \\
\hline Sim & 328 & 32,1 & $29,3-35,1$ \\
\hline \multicolumn{4}{|l|}{ Consumo de tabaco } \\
\hline Não & 920 & 89,9 & $87,9-91,6$ \\
\hline Sim & 103 & 10,1 & $8,4-12,1$ \\
\hline \multicolumn{4}{|l|}{ Consumo de frutas } \\
\hline Inadequado & 678 & 66,6 & $63,6-69,4$ \\
\hline Adequado & 340 & 33,4 & $30,6-36,4$ \\
\hline \multicolumn{4}{|l|}{ Consumo de salada e legumes crus } \\
\hline Inadequado & 589 & 57,6 & $54,5-60,6$ \\
\hline Adequado & 434 & 42,4 & $39,4-45,5$ \\
\hline \multicolumn{4}{|c|}{ Consumo de alimentos ultraprocessados } \\
\hline Não & 620 & 60,6 & $57,6-63,6$ \\
\hline Sim & 403 & 39,4 & $36,4-42,4$ \\
\hline
\end{tabular}

(continua) 
Tabela 1 (continuação)

\begin{tabular}{lccc}
\hline Variável & $\mathbf{n}$ & $\%$ & IC95\% \\
\hline $\begin{array}{l}\text { Percepção do consumo de sal } \\
\quad \text { Baixo/Muito baixo }\end{array}$ & 186 & 18,2 & $15,9-20,7$ \\
$\quad$ Adequado & 668 & 65,2 & $62,3-68,1$ \\
$\quad$ Alto/Muito alto & 170 & 16,6 & $14,4-19,0$ \\
Prática de atividade física & & & \\
$\quad$ Ativo & 646 & 63,2 & $60,2-66,1$ \\
$\quad$ Inativo & 375 & 36,7 & $33,9-39,8$ \\
Saúde/Doença & & & \\
IMC & 523 & 51,1 & $48,1-54,2$ \\
$\quad$ Baixo peso/Eutrófico & 365 & 35,7 & $32,8-38,7$ \\
$\quad$ Sobrepeso & 135 & 13,2 & $11,3-15,4$ \\
$\quad$ Obesidade & & & $69,8-75,3$ \\
Circunferência da cintura & 744 & 72,7 & $24,7-30,2$ \\
$\quad$ Adequada & 280 & 27,3 &
\end{tabular}

IC95\%: intervalo de 95\% de confiança.

* n: número absoluto (pode variar em relação às perdas e os não respondentes);

** Valor do salário mínimo em 2017 no Brasil: R\$937,00.

Foram classificados como hipertensos 28,6\% (IC95\%: 25,9-31,5) dos trabalhadores homens.

Quanto ao estilo de vida desta população, 32,1\% faziam consumo abusivo de álcool e 10,1\% consumiam tabaco. Foi observado que a maioria consumia inadequadamente frutas, saladas e legumes crus. Cerca de $40 \%$ dos entrevistados substituíam as refeições principais por alimentos ultraprocessados e a maioria considerava o seu consumo de sal como adequado (65,2\%). Mais da metade da população foi classificada com ativa fisicamente. Quanto ao estado nutricional, mais de $40 \%$ estava com sobrepeso ou obesidade e $27,3 \%$ apresentaram circunferência da cintura aumentada ou muito aumentada (Tabela 1).

Foi observada em análise bivariada (Tabela 2) uma maior prevalência de hipertensão arterial entre os homens trabalhadores com 40 anos ou mais, casados ou com companheira(o), que consumiam tabaco, que relataram elevado consumo de sal, classificados em inativos fisicamente, com excesso de peso e que apresentaram circunferência da cintura aumentada ou muito aumentada.

A média da percepção de qualidade de vida dos hipertensos foi menor (30,9; desvio padrão DP: $\pm 3,50)$ do que a dos não hipertensos $(31,2$; DP: $\pm 3,51)$, porém, sem significância estatística (valor de $\mathrm{p}=0,14)$.

$\mathrm{Na}$ análise multivariada hierárquica (Tabela 3), foi observada associação da idade de 40 anos ou mais, raça autodeclarada preta e renda maior ou igual à três salários mínimos com uma maior prevalência de hipertensão arterial. Para variáveis modificáveis relacionadas ao estilo de vida, o consumo abusivo de álcool ( $R P=1,24$; IC95\%: 1,02-1,52), consumo de tabaco ( $R P=1,34$; IC95\%: 1,04-1,72), percepção do consumo elevado de sal $(R P=1,64$; IC95\%: 1,20-2,24) e ser fisicamente inativo $(R P=$ 1,23; IC95\%: 1,01-1,49) foram associados positivamente com a hipertensão arterial. Trabalhadores com sobrepeso ou obesidade apresentaram prevalência de hipertensão arterial em mais de 60\%.

Através das razões de prevalência ajustadas obtidas na análise multivariada, foram calculadas as FAP (Tabela 4) para as variáveis de estilo de vida e saúde que se mantiveram associadas no modelo final. Maiores FAP foram observadas para consumo de tabaco, consumo abusivo de álcool, com percepção de consumo elevado de sal, inatividade física e com sobrepeso e obesidade. 
Tabela 2

Análise bivariada da hipertensão arterial na população de trabalhadores homens de um município do Nordeste do Brasil, $2017-2018$.

\begin{tabular}{|c|c|c|c|c|}
\hline Variável & Prevalência de hipertensão arterial (\%) & RP & IC95\% & Valor de $p$ \\
\hline \multicolumn{5}{|l|}{ Sociodemográficas } \\
\hline Idade (anos) & & & & $<0,001$ \\
\hline $18-29$ & 20,4 & 1,00 & & \\
\hline 30-39 & 24,5 & 1,20 & $0,91-1,58$ & \\
\hline 40 ou mais & 44,3 & 2,18 & $1,69-2,80$ & \\
\hline Raça & & & & 0,063 \\
\hline Branca & 27,4 & 1,00 & & \\
\hline Preta & 31,8 & 1,16 & $0,86-1,57$ & \\
\hline Amarela & 7,1 & 0,26 & $0,06-1,01$ & \\
\hline Parda & 28,1 & 1,03 & $0,79-1,33$ & \\
\hline Indígena & 41,2 & 1,50 & $0,81-2,77$ & \\
\hline Estado civil & & & & 0,020 \\
\hline Solteiro & 22,3 & 1,00 & & \\
\hline Casado ou com companheira(o) & 31,0 & 1,39 & $1,09-1,77$ & \\
\hline Separado/Viúvo & 31,8 & 1,42 & $0,94-2,15$ & \\
\hline Renda familiar (salários mínimos) * & & & & 0,195 \\
\hline$<2$ & 25,9 & 1,00 & & \\
\hline $2<3$ & 27,5 & 1,06 & $0,82-1,37$ & \\
\hline$\geq 3$ & 31,8 & 1,23 & $0,98-1,55$ & \\
\hline Anos de estudo (anos) & & & & 0,517 \\
\hline $0-8$ & 26,8 & 1,00 & & \\
\hline $9-11$ & 31,0 & 1,16 & $0,86-1,55$ & \\
\hline$\geq 12$ & 27,7 & 1,03 & $0,79-1,35$ & \\
\hline Turno de trabalho & & & & 0,935 \\
\hline Diurno & 28,3 & 1,00 & & \\
\hline Diurno/Noturno & 28,6 & 1,01 & $0,79-1,29$ & \\
\hline Cargos de trabalho & & & & 0,638 \\
\hline Não braçal & 27,8 & 1,00 & & \\
\hline Braçal & 29,1 & 1,05 & $0,86-1,28$ & \\
\hline \multicolumn{5}{|l|}{ Estilo de vida } \\
\hline Consumo abusivo de álcool & & & & 0,056 \\
\hline Não & 26,8 & 1,00 & & \\
\hline $\operatorname{sim}$ & 32,6 & 1,22 & $1,00-1,48$ & \\
\hline Consumo de tabaco & & & & 0,008 \\
\hline Não & 27,4 & 1,00 & & \\
\hline $\operatorname{sim}$ & 39,8 & 1,45 & $1,12-1,88$ & \\
\hline Consumo de frutas & & & & 0,228 \\
\hline Adequado & 26,2 & 1,00 & & \\
\hline Inadequado & 29,8 & 1,14 & $0,92-1,40$ & \\
\hline Consumo de salada e legumes crus & & & & 0,473 \\
\hline Adequado & 29,7 & 1,00 & & \\
\hline Inadequado & 27,7 & 0,93 & $0,77-1,13$ & \\
\hline Consumo de alimentos ultraprocessados & & & & 0,674 \\
\hline Não & 28,1 & 1,00 & & \\
\hline $\operatorname{sim}$ & 29,3 & 1,04 & $0,86-1,27$ & \\
\hline
\end{tabular}

(continua) 
Tabela 2 (continuação)

\begin{tabular}{|c|c|c|c|c|}
\hline Variável & Prevalência de hipertensão arterial (\%) & $\mathbf{R P}$ & IC95\% & Valor de $p$ \\
\hline Percepção do consumo de sal & & & & 0,005 \\
\hline Baixo/Muito baixo & 25,8 & 1,00 & & \\
\hline Adequado & 26,8 & 1,04 & $0,79-1,37$ & \\
\hline Alto/Muito alto & 38,8 & 1,50 & $1,11-2,04$ & \\
\hline Prática de atividade física & & & & 0,016 \\
\hline Ativo & 26,0 & 1,00 & & \\
\hline Inativo & 33,1 & 1,27 & $1,05-1,54$ & \\
\hline \multicolumn{5}{|l|}{ Saúde/Doença } \\
\hline Índice de massa corporal & & & & $<0,001$ \\
\hline Baixo peso/Eutrófico & 19,3 & 1,00 & & \\
\hline Sobrepeso & 34,5 & 1,79 & $1,43-2,24$ & \\
\hline Obesidade & 48,9 & 2,53 & $1,98-3,24$ & \\
\hline Circunferência da cintura & & & & $<0,001$ \\
\hline Adequada & 22,9 & 1,00 & & \\
\hline Aumentada/Muito aumentada & 43,9 & 1,92 & $1,59-2,32$ & \\
\hline
\end{tabular}

IC95\%: intervalo de 95\% de confiança; RP: razão de prevalência.

* Valor do salário mínimo em 2017 no Brasil: R\$937,00.

Tabela 3

Análise multivariada da hipertensão arterial na população de trabalhadores homens de um município do Nordeste do Brasil, 2017-2018.

\begin{tabular}{|c|c|c|c|c|c|c|}
\hline \multirow[t]{2}{*}{ Variáveis } & \multicolumn{2}{|c|}{ Modelo 1} & \multicolumn{2}{|c|}{ Modelo 2} & \multicolumn{2}{|c|}{ Modelo 3} \\
\hline & $\mathbf{R P}$ & IC95\% & $\mathbf{R P}$ & IC95\% & $\mathbf{R P}$ & IC95\% \\
\hline \multicolumn{7}{|c|}{ Sociodemográficas } \\
\hline \multicolumn{7}{|l|}{ Idade (anos) } \\
\hline $18-29$ & 1,00 & & 1,00 & & 1,00 & \\
\hline $30-39$ & 1,12 & $0,85-1,47$ & 1,09 & $0,83-1,44$ & 1,00 & $0,76-1,31$ \\
\hline 40 ou mais & 2,01 & $1,56-2,60$ * & 1,92 & $1,48-2,48$ * & 1,59 & $1,22-2,06$ ** \\
\hline \multicolumn{7}{|l|}{ Raça } \\
\hline Branca & 1,00 & & 1,00 & & 1,00 & \\
\hline Preta & 1,36 & $1,01-1,83 * *$ & 1,41 & $1,06-1,88 * *$ & 1,37 & $1,03-1,81 * *$ \\
\hline Amarela & 0,34 & $0,09-1,30$ & 0,30 & $0,08-1,18$ & 0,32 & $0,08-1,22$ \\
\hline Parda & 1,06 & $0,81-1,38$ & 1,10 & $0,85-1,41$ & 1,10 & $0,85-1,36$ \\
\hline Indígena & 1,64 & $0,95-2,84$ & 1,68 & $0,99-2,82$ & 1,65 & $0,90-3,01$ \\
\hline \multicolumn{7}{|c|}{ Renda familiar (salários mínimos) *** } \\
\hline$<2$ & 1,00 & & 1,00 & & 1,00 & \\
\hline $2<3$ & 1,14 & $0,88-1,47$ & 1,12 & $0,86-1,44$ & 1,03 & $0,80-1,32$ \\
\hline$\geq 3$ & 1,26 & $1,01-1,59$ * & 1,22 & $0,97-1,54$ & 1,08 & $0,85-1,36$ \\
\hline
\end{tabular}

(continua) 
Tabela 3 (continuação)

\begin{tabular}{|c|c|c|c|c|c|c|}
\hline \multirow[t]{2}{*}{ Variáveis } & \multicolumn{2}{|c|}{ Modelo 1} & \multicolumn{2}{|c|}{ Modelo 2} & \multicolumn{2}{|c|}{ Modelo 3} \\
\hline & $\mathbf{R P}$ & IC95\% & $\mathbf{R P}$ & IC95\% & $\mathbf{R P}$ & IC95\% \\
\hline \multicolumn{7}{|l|}{ Estilo de vida } \\
\hline \multicolumn{7}{|c|}{ Consumo abusivo de álcool } \\
\hline Não & & & 1,00 & & 1,00 & \\
\hline Sim & & & 1,24 & $1,02-1,52$ ** & 1,13 & 0,93-1,39 \\
\hline \multicolumn{7}{|l|}{ Consumo de tabaco } \\
\hline Não & & & 1,00 & & 1,00 & \\
\hline Sim & & & 1,34 & $1,04-1,72 * *$ & 1,53 & $1,18-1,99 *$ \\
\hline \multicolumn{7}{|c|}{ Percepção do consumo de sal } \\
\hline Baixo/Muito baixo & & & 1,00 & & 1,00 & \\
\hline Adequado & & & 1,18 & $0,89-1,57$ & 1,19 & $0,90-1,57$ \\
\hline Alto/Muito alto & & & 1,64 & $1,20-2,24 \#$ & 1,53 & $1,12-2,08$ \# \\
\hline \multicolumn{7}{|l|}{ Prática de atividade física } \\
\hline Ativo & & & 1,00 & & 1,00 & \\
\hline Inativo & & & 1,23 & $1,01-1,49 * *$ & 1,23 & $1,02-1,49 * *$ \\
\hline \multicolumn{7}{|l|}{ Saúde/Doença } \\
\hline \multicolumn{7}{|l|}{ Índice de massa corporal } \\
\hline Baixo peso/Eutrófico & & & & & 1,00 & \\
\hline Sobrepeso & & & & & 1,64 & $1,29-2,09$ * \\
\hline Obesidade & & & & & 2,14 & $1,64-2,78$ * \\
\hline Critério Akaike & & .599 & & 36.268 & & 18.612 \\
\hline
\end{tabular}

IC95\%: intervalo de 95\% de confiança; Modelo 1: ajustado entre as variáveis sociodemográficas; Modelo 2: ajustado entre as variáveis sociodemográficas e de estilo de vida; Modelo 3: ajustado entre as variáveis sociodemográficas, estilo de vida e saúde/doença; RP: razão de prevalência.

$* p<0,001$;

$* * \mathrm{p}<0,05$;

*** Valor do salário mínimo em 2017 no Brasil: $\mathrm{R} \$$ 937,00;

$\# p<0,01$.

\section{Tabela 4}

Frações atribuíveis para fatores associados modificáveis entre os expostos em uma população de trabalhadores homens de um município do Nordeste do Brasil, 2017-2018.

\begin{tabular}{lc}
\hline Variáveis & Fração atribuível (\%) \\
\hline Consumo abusivo de álcool & 7,07 \\
Consumo de tabaco & 3,55 \\
Percepção do consumo de sal alto/muito alto & 8,79 \\
Inatividade física & 7,94 \\
Índice de massa corporal & \\
$\quad$ Sobrepeso & 16,78 \\
$\quad$ Obeso & 12,00 \\
\hline
\end{tabular}




\section{Discussão}

Com este estudo foi possível identificar a presença de DCNT prevalente em população masculina de trabalhadores no período em que foram realizar consulta periódica do trabalho. Variáveis sociodemográficas apresentaram associações com o evento e foram inseridas como importantes fatores de ajustes. Variáveis modificáveis, passíveis de intervenção, mantiveram-se associadas no modelo ajustado. O consumo abusivo de álcool, consumo de tabaco, percepção de consumo elevado de sal, a inatividade física e o sobrepeso/obesidade associaram-se a uma maior prevalência de hipertensão arterial nesta população.

A hipertensão arterial tem se destacado como DCNT de alta prevalência em populações masculinas 11,37,38 e de trabalhadores 12,39,40, evidenciando a necessidade de identificação precoce dos agravos crônicos para planejar ações específicas voltadas para a população acometida.

Na presente pesquisa, os trabalhadores com 40 anos ou mais apresentaram uma maior prevalência da hipertensão arterial. O envelhecimento está associado a alterações fisiológicas e outras comorbidades $11,39,40$, o que promove maior procura aos serviços de saúde, aumentando o monitoramento de doenças assintomáticas ${ }^{41}$. No entanto, observou-se neste estudo um percentual de trabalhadores entre 30-39 anos também acometidos por hipertensão arterial, ou seja, o trabalho pode exercer possíveis efeitos negativos precoces na saúde 42 . O status econômico também pode estar associado à maior prevalência de hipertensão arterial, conforme resultados deste estudo. Isso pode ser explicado porque a maior renda pode promover maior acesso à informação e aumento da demanda por serviços de saúde 41 , ampliando as oportunidades de diagnóstico de doenças crônicas.

Os trabalhadores que autodeclaram cor da pele preta apresentaram associação positiva com a hipertensão arterial. Observa-se uma morbimortalidade maior em decorrência da hipertensão arterial entre pessoas de cor da pele preta 32,38,43. O Brasil apresenta taxas de morbimortalidade fortemente afetadas por desigualdades sociais, causando uma distribuição desigual de DCNT como hipertensão arterial 4,11. A Região Nordeste é historicamente identificada por apresentar um grande contingente de pretos e pardos e menores condições de renda, saúde e educação, tendo vários grupos populacionais em situações de vulnerabilidade 11,32. Os contextos em que os grupos populacionais estão inseridos podem determinar mais a presença de DCNT do que as diferenças genéticas observadas entre raças 43,44. A redução das desigualdades sociais pode ampliar a prevenção e o controle de DCNT no Brasil, principalmente em regiões mais afetadas pelas iniquidades.

Neste estudo, não foi observada associação significativa entre turno de trabalho e hipertensão arterial, possivelmente devido à baixa frequência de trabalho noturno na amostra estudada. Resultado semelhante foi observado em uma coorte realizada no Brasil com trabalhadores de um hospital público 9. Em contrapartida, em uma coorte de trabalhadores homens, na Espanha, foi observado que a incidência de doenças crônicas ocorreu mais nos que assumiam turnos noturnos de trabalho e isso pode contribuir para a maior prevalência de doenças crônicas devido aos ritmos circadianos alterados, estresse e alterações bioquímicas que ocorrem no organismo 12.

Grupos prioritários para rastreio foram identificados como aqueles que apresentaram fatores modificáveis associados ao evento estudado. Os que relataram consumir álcool de maneira abusiva apresentaram maior prevalência da hipertensão arterial. Conforme estudo prévio, esse hábito foi associado à insegurança no trabalho, restrições profissionais, insatisfações no ambiente laboral e situações de estresse 7 . O consumo abusivo de álcool pode aumentar a prevalência de doenças e limitações funcionais, além de estar relacionado a eventos violentos 24 .

Os trabalhadores que faziam o consumo de tabaco apresentaram maior prevalência de hipertensão arterial. Em estudo realizado na Bolívia envolvendo trabalhadores, observou-se que os mais vulneráveis ao uso do tabaco estavam mais expostos a estresse no local de trabalho 45 , mostrando que o uso de substâncias ilícitas por vezes pode estar associado à necessidade de relaxamento 7 . O uso do tabaco entre trabalhadores pode gerar aumento das taxas de absenteísmo, devido a maior possibilidade de surgimento de doenças 40,46. Em contrapartida, se houvesse redução ou eliminação desse hábito, a prevalência da DCNT estudada poderia ser reduzida em 3,55\% de acordo com a FAP calculada.

Observa-se no decorrer das décadas uma preocupação em promover ações e campanhas para redução dos efeitos deletérios das DCNT no ambiente de trabalho, com resultados positivos 1,2,8,12. Portanto, a intensificação das campanhas para redução ou cessação do consumo de taba- 
co é de suma importância, pois podem promover a descontinuação da prática e melhorar o estilo de vida dos trabalhadores.

Para o perfil de alimentação, os participantes que declararam consumir sal em elevada quantidade tiveram maior prevalência de hipertensão arterial, e este consumo mostrou um resultado importante de FAP que pode ser utilizado nas estratégias de promoção de alimentação saudável no ambiente de trabalho. Já foi observado na literatura uma maior ingestão de sódio entre os trabalhadores homens 8 .

A maior parte do sal consumido por trabalhadores provém de alimentos processados e de refeições feitas fora de casa, devido a uma rotina acelerada 8, características da população do presente trabalho. Hábitos alimentares não saudáveis podem favorecer o aparecimento de comorbidades, aumentar os índices de absenteísmo e consequentemente diminuir o tempo exercendo atividade produtiva 8,28. Atividades de educação nutricional que vão desde orientações específicas em grupos a um planejamento adequado de cardápios devem ser realizadas nos espaços coletivos de trabalhos. O espaço laboral pode ser um local promotor de saúde e o trabalhador, um multiplicador de hábitos saudáveis no ambiente familiar e na sociedade.

Quanto à prática de atividade física, observa-se no ambiente de trabalho uma tendência de redução do gasto energético e aumento da inatividade física. A atividade física quando regular, pode contribuir para redução dos níveis pressóricos, no entanto, há uma baixa adesão entre os hipertensos, especialmente trabalhadores, devido à jornada laboral extensa, tipo de atividade realizada e ao cansaço físico 47,48 . Neste estudo, foi observado que se reduzisse ou eliminasse a inatividade física entre os trabalhadores homens, a prevalência da DCNT reduziria em 7,94\%. As novas diretrizes da OMS (2020) sobre atividade física e comportamento sedentário reforçam o valor das atividades físicas de qualquer duração e os benefícios de atividade física de intensidade leve como um substituto para tempo sedentário 49. Portanto, promover intervenções que estimulem a redução do sedentarismo, como o incentivo a atividades de intensidade leve e pequenas séries de atividades, como caminhar ou subir escadas, tanto nos ambientes laborais como nos ambientes extralaborais, podem contribuir na promoção da saúde dos trabalhadores e redução de complicações em longo prazo.

O elevado peso corporal pode ser considerado um fator de risco para o desenvolvimento de DCNT 9,31 no caso a hipertensão arterial, contribuindo com o aumento de até $30 \%$ dos casos ${ }^{50}$. Neste estudo foi observada uma maior presença deste estado nutricional desfavorável entre os trabalhadores classificados como hipertensos. De acordo com os resultados a eliminação ou redução do sobrepeso e obesidade reduziria em mais de $28 \%$ a prevalência da hipertensão arterial neste grupo. Esses achados indicam que estilos de vida não saudáveis, muitas vezes fomentados pelo próprio contexto do trabalho, podem afetar negativamente a saúde dos trabalhadores, repercutindo em aumento do peso corporal com consequente surgimento de doenças crônicas 8,9,39.

Prevenir o surgimento da hipertensão arterial ou reduzir níveis de pressóricos entre os hipertensos no local de trabalho envolve primeiramente conhecer o perfil dos trabalhadores, a manifestação da doença, bem como suas complicações e, posteriormente, contribuir com um ambiente favorável para mudanças de hábitos de vida e adesão a tratamentos. Essas mudanças podem ser promovidas por meio de ações individualizadas, de acordo com demandas específicas de cada trabalhador, e também por meio de ações coletivas, de modo a ampliar o campo de ação, podendo ser mantidas a longo prazo 51 .

A hipertensão arterial quando não tratada adequadamente causa complicações e, em muitos casos, levam o indivíduo a requerer cuidados médicos de alto custo, exigindo uso constante de medicamentos e exames complementares periódicos, o que pode causar impactos diretos no ambiente familiar e laboral, devido à perda da produtividade e do absenteísmo. Por isso, estratégias que viabilizem, no ambiente de trabalho, medidas de prevenção de doença e promoção da saúde podem antecipar precocemente a situação de risco e danos à saúde do trabalhador 51 . Importante lembrar que, conforme a Convenção 161 52, da Organização Internacional do Trabalho (OIT), em seu artigo 12, as ações de promoção da saúde em relação com o trabalho não deverão significar ônus financeiro aos trabalhadores, e, na medida do possível, deverá realizar-se durante as horas de trabalho.

Programas de qualidade de vida no trabalho não se enquadram nos conceitos e obrigações do empregador. Entretanto, é sabido que tais programas contribuem para a melhoria da produtividade dos colaboradores, que ficam mais dispostos e motivados, além de aptos emocionalmente para entregar grandes resultados. Equipes insatisfeitas tornam-se improdutivas, estão mais propensas a erros e costumam envolver-se em conflitos 53. 
Importante citar ainda as alterações no processo e na organização do trabalho observadas em todo o mundo, que intensificam o ritmo e ampliam as jornadas, favorecendo o surgimento de trabalhos informais, sem garantias trabalhistas 54, tornando-se uma ameaça à saúde e ao bem-estar do trabalhador 53 .

O cálculo da FAP realizado no presente trabalho pode fornecer informações importantes sobre o potencial impacto de programas de prevenção e intervenções em saúde, sendo útil para gestores, visto que essas informações podem ser exploradas pelos órgãos de atenção à saúde do trabalhador, de cada empresa, a fim de atuarem, visando as particularidades de cada local de trabalho e perfil do trabalhador. Adicionado aos serviços oferecidos pelas empresas durante os turnos de trabalho, tem-se também iniciado em algumas localidades do Brasil o funcionamento estendido de algumas unidades de saúde da família (USF). Desde a publicação da Portaria no 930/2019 55, foi ampliada a cobertura de algumas USF, o que proporciona maior acesso de usuários nas ações e nos serviços promovidos.

O presente estudo apresenta algumas limitações. O delineamento transversal dificulta o estabelecimento de relações causa e efeito entre algumas variáveis. Em alguns momentos da discussão a comparabilidade com outros estudos foi dificultada devido à escassez de estudos envolvendo populações de trabalhadores homens. As medidas objetivas da pressão arterial foram realizadas em um mesmo momento. O diagnóstico clínico preconiza pelo menos duas coletas em momentos diferentes 56, porém, estudos epidemiológicos têm sido importantes para a realização de rastreio da hipertensão arterial e a geração de indicadores, e diferentes estudos de base populacional são realizados com a mesma metodologia abordada pelo presente trabalho 4,11,38,40. Alguns fatores de risco que ameaçam a saúde do trabalhador e não estão associados ao estilo de vida, como clima organizacional, insegurança no trabalho, restrições profissionais, insatisfação no emprego, exposição a ruídos, dentre outras, não foram testados neste estudo e podem ser alvo de futuras investigações.

A presente pesquisa também possui uma amostra robusta, com um pequeno erro amostral. Todos os dados foram coletados seguindo protocolos previamente estabelecidos, e os pesquisadores foram treinados, reduzindo a possibilidade de erros de coleta de dados.

\section{Considerações finais}

Observou-se neste estudo que hábitos e comportamentos não saudáveis estavam mais presentes entre aqueles acometidos pela DCNT estudada. A população de trabalhadores tem sido vista como um público de difícil acesso para rastreio da situação de saúde, devido a carga horária laboral extensa, muitas vezes incompatíveis com os horários disponíveis pelos serviços de saúde. Somado a isso temse a menor preocupação com saúde pela população masculina. Neste cenário, uma triagem precoce de DCNT, seja no local de trabalho ou em serviços públicos de atenção à saúde, oportuniza a identificação dos principais fatores de risco cardiovasculares e propõe estratégias de promoção, proteção e recuperação da saúde entre aos trabalhadores, tais como acompanhamento individual e em grupo, assim como ações de sensibilização para modificar hábitos considerados não saudáveis.

O presente estudo apresenta relevância por estudar a uma DCNT importante em uma população específica e que necessita de atenção diferenciada. Poucos estudos abordam as questões acerca da saúde dos trabalhadores, especificamente homens, por ser um grupo populacional com restrições de tempo, disponibilidade reduzida para participação em pesquisas e pouco interesse em priorizar sua própria saúde. 


\section{Colaboradores}

Todos os autores contribuíram na concepção e projeto do estudo, análise e interpretação dos dados, na redação do artigo e revisão crítica relevante do conteúdo intelectual, na aprovação final da versão a ser publicada, e são responsáveis por todos os aspectos do trabalho na garantia da exatidão e integridade de qualquer parte da obra

\section{Informações adicionais}

ORCID: Tamyres Araújo Andrade Donato (00000003-0930-1101); Roberta Mendes Abreu Silva (0000-0001-5057-1643); Amanda Cristina de Souza Andrade (0000-0002-3366-4423); Clávdia Nicolaevna Kochergin (0000-0002-3272-3030); Danielle Souto de Medeiros (0000-0002-2480-8990); Daniela Arruda Soares (0000-0002-4801-1011); José Andrade Louzado (0000-0003-4446-7051); Kelle Oliveira Silva (0000-0003-2041-1088); Matheus Lopes Cortes (0000-0002-7804-7787); Sóstenes Mistro (0000-0001-5840-820X); Welma Wildes Cunha Coelho Amorim (0000-0001-6964-892X); Márcio Galvão Guimarães de Oliveira (0000-00015281-7889); Vanessa Moraes Bezerra (0000-00015333-2875).

\section{Referências}

1. Put VD, Anne C, Lippe VD. Work environment and worksite health promotion in nine European countries. J Occup Environ Med 2020; 62:272-8.

2. Legorreta AP, Schaff SR, Leibowitz AN, van Meijgaard J. Measuring the effects of screening programs in asymptomatic employees: detection of hypertension through worksite screenings. J Occup Environ Med 2015; 57:682-6.

3. World Health Organization. A global brief on hypertension - silent killer, global public health crisis. Geneva: World Health Organization; 2013.

4. World Health Organization. Global status report on noncommunicable diseases 2010. Geneva: World Health Organization; 2010.

5. Risso-Gill I, Balabanova D, Majid F, Ng KK, Yusoff K, Mustapha F, et al. Understanding the modifiable health systems barriers to hypertension management in Malaysia: a multimethod health systems appraisal approach. BMC Health Serv Res 2015; 15:254.

\section{Agradecimentos}

Esta pesquisa faz parte do projeto HealthRise, em parceria com a Medtronic Foundation, Abt Associates e o Institute for Health Metrics and Evaluation (IHME). Agradecemos a todos os trabalhadores participantes pela disponibilidade em nos receber. Agradecemos a Coordenação de Aperfeiçoamento de Pessoal de Nível Superior (CAPES; código de financiamento 001), pelo apoio no financiamento.
6. Azizova T, Briks K, Bannikova M, Grigoryeva E. Hypertension incidence risk in a cohort of Russian workers exposed to radiation at the Mayak Production Association over prolonged periods. Hypertension 2019; 73:1174-84.

7. Boeuf-Cazou O, Lapeyre-Mestre M, Niezborala M, Montastruc JL. Profile of psychoactive substances consumption in workplace. Therapie 2011; 66:155-65.

8. Reinaldo JM, Resende AS, Sant Anna MSL. Prevalência de hipertensão arterial e avaliação da ingestão de sódio em uma Unidade de Alimentação e Nutrição do Estado de Sergipe/ Brasil. Revista da Associação Brasileira de Nutrição 2017; 8:58-63.

9. Montzel DRB, Costa BVL, Silva FM. Ganho de peso por década entre trabalhadores de um hospital público: estudo de coorte histórica. Ciênc Saúde Colet 2019; 24:2453-60.

10. World Health Organization. Global action plan for the prevention and control of NCDs 2013-2020. Geneva: World Health Organization; 2013. 
11. Malta DC, Gonçalves RPF, Machado IE, Freitas MIF, Azeredo C, Szwarcwald CL. Prevalence of arterial hypertension according to different diagnostic criteria, National Health Survey. Rev Bras Epidemiol 2018; 21:e180021.

12. Aguilar-Palacio I, Malo S, Feja C, Lallana M, Leon-Latre M, Casasnovas JA, et al. Risk factors control for primary prevention of cardiovascular disease in men: evidence from the Aragon Workers Health Study (AWHS). PLoS One 2018; 13:e0193541.

13. Flor LS, Wilson S, Bhatt P, Bryant M, Burnett A, Camarda JN, et al. Community-based interventions for detection and management of diabetes and hypertension in underserved communities: a mixed-methods evaluation in Brazil, India, South Africa and the USA. BMJ Global Health 2020; 5:e001959.

14. Instituto Brasileiro de Geografia e Estatística. Vitória da Conquista, panorama. https://cida des.ibge.gov.br/brasil/ba/vitoria-da-conquis ta/panorama (acessado em 18/Ago/2018).

15. Departamento Nacional, Serviço Social da Indústria. Estilo de vida e hábitos de lazer dos trabalhadores das indústrias brasileiras: sumário executivo. Brasília: Departamento Nacional, Serviço Social da Indústria; 2009.

16. Instituto Brasileiro de Geografia e Estatística. Questionário Pesquisa Nacional de Saúde. https://www.ibge.gov.br/estatisti cas/sociais/saude/9160-pesquisa-nacionalde-saude.html? =\&t=o-que-e (acessado em 13/ Jun/2018).

17. Matsudo S, Araújo T, Matsudo V, Andrade D, Andrade E, Oliveira LC, et al. Questionário internacional de atividade física (IPAQ): estudo de validade e reprodutibilidade no Brasil. Rev Bras Ativ Fís Saúde 2012; 10:41-50.

18. Pereira M, Melo C, Gameiro S, Canavarro MC. Estudos psicométricos da versão em Português Europeu do índice de qualidade de vida EUROHIS-QOL-8. Laboratório de Psicologia 2011; 9:109-23.

19. Topouchian JA, El Assaad MA, Orobinskaia LV, El Feghali RN, Asmar RG. Validation of two automatic devices for self-measurement of blood pressure according to the International Protocol of the European Society of Hypertension: the Omron M6 (HEM-7001-E) and the Omron R7 (HEM 637-IT). Blood Press Monit 2006; 11:165-71.

20. Sociedade Brasileira de Cardiologia. 7a Diretriz Brasileira de Hipertensão Arterial. Arq Bras Cardiol 2016; 107:1-83.

21. Kirchhof ALC, Magnago TSBS, Camponogara S, Griep RH, Tavares JP, Prestes FC, et al. Condições de trabalho e características sóciodemográficas relacionadas à presença de distúrbios psíquicos menores em trabalhadores de enfermagem. Texto Contexto Enferm 2009; 18:215-23.
22. Aguiar OB, Valente JG, Fonseca MJM. Descrição sócio-demográfica, laboral e de saúde dos trabalhadores do setor de serviços de alimentação dos restaurantes populares do estado do Rio de Janeiro. Rev Nutr 2010; 23:969-82.

23. Ministério do Trabalho e Emprego. Classificação Brasileira de Ocupações. https://www. ocupacoes.com.br/ (acessado em 25/Jul/2018).

24. Oliveira JL, Souza J. Factors associated with alcohol consumption among public maintenance workers. Acta Paul Enferm 2018; 31:17-24.

25. World Health Organization. Global status report on alcohol and health 2014. Geneva: World Health Organization; 2014.

26. Departamento de Vigilância de Doenças e Agravos Não Transmissíveis e Promoção da Saúde, Secretaria de Vigilância em Saúde, Ministério da Saúde. Vigitel Brasil 2015: vigilância de fatores de risco e proteção para doenças crônicas por inquérito telefônico. Estimativas sobre frequência e distribuição sociodemográfica de de fatores de risco e proteção para doenças crônicas nas capitais dos 26 estados brasileiros e no Distrito Federal em 2015. Brasília: Ministério da Saúde; 2017.

27. Departamento de Atenção Básica, Secretaria de Atenção à Saúde, Ministério da Saúde. Guia alimentar para a população brasileira. 2a Ed. Brasília: Ministério da Saúde; 2014.

28. Oliveira MM, Malta DC, Santos MAS, Oliveira TP, Nilson EAF, Claro RM. Self-reported high salt intake in adults: data from the National Health Survey, Brazil, 2013. Epidemiol Serv Saúde 2015; 24:249-56.

29. World Health Organization. Global recommendations on physical activity for health. Geneva: World Health Organization; 2010.

30. Hallal PC, Victora CG, Wells JC, Lima RC. Physical inactivity: prevalence and associated variables in Brazilian adults. Med Sci Sports Exerc 2003; 35:1894-900.

31. World Health Organization. Obesity: preventing and managing the global epidemic. Geneva: World Health Organization; 2000.

32. Bezerra VM, Andrade ACS, César CC, Caiaffa WT. Comunidades quilombolas de Vitória da Conquista, Bahia, Brasil: hipertensão arterial e fatores associados. Cad Saúde Pública 2013; 29:1889-902.

33. Victora CG, Huttly SR, Fuchs SC, Olinto MTA. The role of conceptual frameworks in epidemiological analysis: a hierarchical approach. Int J Epidemiol 1997; 26:224-7.

34. Costa JSD, Barcellos FC, Sclowitz ML, Sclowitz IKT, Castanheira M, Olinto MTA, et al. Prevalência de hipertensão arterial em adultos e fatores associados: um estudo de base populacional urbana em Pelotas, Rio Grande do Sul, Brasil. Arq Bras Cardiol 2007; 88:59-65.

35. Kadane JB, Lazar NA. Métodos e critérios de seleção de modelos. J Am Statist Assoc 2004; 99:279-90. 
36. Rezende LFM, Eluf-Neto, J. Fração atribuível populacional: planejamento de ações de prevenção de doenças no Brasil. Rev Saúde Pública 2016; 50:30.

37. Nyuyki CK, Ngufor G, Mbeh G, Mbanya JC. Epidemiology of hypertension in Fulani indigenous populations-age, gender and drivers. J Health Popul Nutr 2017; 36:35.

38. Tripathy JP, Thakur JS, Jeet G, Chawla S, Jain $S$. Alarmingly high prevalence of hypertension and pre-hypertension in North India-results from a large cross-sectional STEPS survey. PLoS One 2017; 12:e0188619.

39. Itani O, Kaneita Y, Tokiya M, Jike M, Murata A, Nakagome S, et al. Short sleep duration, shift work, and actual days taken off work are predictive life-style risk factors for newonset metabolic syndrome: a seven-year cohort study of 40,000 male workers. Sleep Med 2017; 39:87-94.

40. Oshiro ML, Ferreira JS, Oshiro E. Hipertensão arterial em trabalhadores da estratégia saúde da família. Rev Bras Ciênc Saúde 2013; 11:20-8.

41. Malta DC, Santos NB, Perillo RD, Szwarcwald CL. Prevalence of high blood pressure measured in the Brazilian population, National Health Survey, 2013. São Paulo Med J 2016; 134:163-70.

42. Tucker P, Harma M, Ojajarvi A, Kivimaki M, Leineweber C, Oksanen T, et al. Associations between shift work and use of prescribed medications for the treatment of hypertension, diabetes, and dyslipidemia: a prospective cohort study. Scand J Work Environ Health 2019; 45:465-74.

43. Chor D, Pinho Ribeiro AL, Carvalho MS, Duncan BB, Andrade Lotufo P, Araújo Nobre A, et al. Prevalence, awareness, treatment and influence of socioeconomic variables on control of high blood pressure: results of the ELSA-Brasil Study. PLoS One 2015; 10:e0127382.

44. Agyemang C, Addo J, Bhopal R, Aikins AG, Stronks K. Cardiovascular disease, diabetes and established risk factors among populations of sub-Saharan African descent in Europe: a literature review. Global Health 2009; 5:7.

45. Arias-Uriona AM, Ordonez JC. Factors involved in job insecurity and their relationship with the health of salaried workers and contract workers in Bolivia. Rev Panam Salud Pública 2018; 42:e98.

46. Fernandes C, Pereira A. Exposure to psychosocial risk factors in the context of work: a systematic review. Rev Saúde Pública 2016; 50:24.
47. Medina C, Janssen I, Barquera S, BautistaArredondo S, Gonzalez ME, Gonzalez C. Occupational and leisure time physical inactivity and the risk of type II diabetes and hypertension among Mexican adults: a prospective cohort study. Sci Rep 2018; 8:5399.

48. Edmunds S, Hurst L, Harvey K. Physical activity barriers in the workplace: an exploration of factors contributing to non-participation in a UK workplace physical activity intervention. Int J Workplace Health Manag 2013; 6:227-40.

49. Ding D, Mutrie N, Bauman A, Pratt M, Hallal PRC, Powell KE. Physical activity guidelines 2020: comprehensive and inclusive recommendations to activate populations. Lancet 2020; 396:1780-2.

50. Associação Brasileira para o Estudo da Obesidade e da Síndrome Metabólica. Diretrizes brasileiras de obesidade. 4a Ed. São Paulo: Associação Brasileira para o Estudo da Obesidade e da Síndrome Metabólica; 2016.

51. Centers for Disease Control and Prevention. Worker productivity|Blood pressure evaluation measures. https://www.cdc.gov/work placehealthpromotion/health-strategies/ blood-pressure/evaluation-measures/workerproductivity.html (acessado em 28/Mar/2020).

52. Organização Internacional do Trabalho. Convenção 161. http://www.trabalhoseguro.com/ OIT/OIT_161_servicos_saude.htm (acessado em 10/Ago/2020).

53. Clein C, Tonello R, Pessa SLR. Influência do ambiente de trabalho na saúde física e emocional do trabalhador: estudo ergonômico em uma fábrica de máquinas industriais. Revista ADMpg Gestão Estratégica 2014; 7:53-9.

54. Gondim AA, Pinheiro JAM, Mendes CF, Neves L. O impacto do processo de precarização laboral em serviços de saúde. Rev SBPH 2018; 21:56-73.

55. Ministério da Saúde. Portaria no 930, de 15 de maio de 2019. Institui o Programa "Saúde na Hora", que dispõe sobre o horário estendido de funcionamento das Unidades de Saúde da Família, altera a Portaria no 2.436/GM/MS, de 2017, a Portaria de Consolidação no 2/GM/ MS, de 2017, a Portaria de Consolidação no 6/GM/MS, de 2017, e dá outras providências. Diário Oficial da União 2019; 17 mai.

56. National Institutes of Health. The Sixth Report of the Joint National Committee on Prevention, Detection, Evaluation, and Treatment of High Blood Pressure. Arch Intern Med 1997; 157:2413-46. 


\section{Abstract}

The study aimed to estimate the prevalence of arterial hypertension as the principal marker of chronic noncommunicable diseases (NCDs) and to identify associated modifiable factors in male workers. Baseline data were used from a longitudinal study with a sample of 1,024 male workers 18 years or older in a municipality in Northeast Brazil. The marker for NCDs was arterial hypertension, defined as systolic pressure $\geq 140 \mathrm{mmHg}$ and/or diastolic pressure $\geq 90 \mathrm{mmHg}$ and/or prior diagnosis of arterial hypertension and/or use of antihypertensive medication. Poisson regression with robust variance was used, adopting hierarchical entry of variables. Population attributable fractions (PAFs) were calculated for the lifestyle variables to measure the impact of modifiable factors on workers' health. Prevalence of hypertension was 28.6\% (95\%CI: 25.9-31.5). Distal factors associated with hypertension were age $>40$ years, black skin color, and family income $\geq 3$ times the monthly minimum wage. Intermediate factors were alcohol abuse, smoking, high self-rated salt intake, and physical inactivity. Proximal factors were overweight and obesity. Calculation of PAFs showed that a reduction or elimination of unhealthy lifestyle habits and behaviors in this population group would reduce the prevalence of the target NCD, hypertension, by 56.1\%. The identification of modifiable factors and the ways they can negatively impact male workers' health allows planning interventions in the workplace itself to reach the largest number of individuals, aimed at reducing the harmful effects of NCDs.

Medical Examination; Diagnosis; Noncommunicable Diseases; Occupational Health; Men

\section{Resumen}

El objetivo fue estimar la prevalencia de hipertensão arterial, como principal marcador de enfermedad crónica no transmisible (ECNT), así como identificar factores modificables asociados, en hombres trabajadores. Se utilizaron datos de la línea de base, procedentes de un estudio longitudinal, con una muestra de 1.024 hombres trabajadores con 18 años o más de un municipio del Nordeste de Brasil. El marcador de ECNT fue la hipertensão arterial, definida por presión arterial sistólica $\geq 140 \mathrm{mmHg}$ y/o presión arterial diastólica $\geq 90 \mathrm{mmHg}$ y/o diagnóstico previo de hipertensão arterial y/o uso de medicamentos antihipertensivos. Se empleó la regresión de Poisson con varianza robusta, adoptando la entrada jerárquica de variables. Se calcularon fracciones atribuibles poblacionales (FAP) en las variables de estilo de vida, a fin de dimensionar el impacto de los factores modificables en la salud de los trabajadores. La prevalencia de la hipertensão arterial en esta población fue de 28,6\% (IC95\%: 25,9-31,5), los factores distales: edad > 40 años, color de piel negra y renta familiar $\geq 3$ salarios minimos; factores intermedios: consumo abusivo de alcohol, consumo de tabaco, percepción de un consumo elevado de sal e inactividad fisica y el factor proximal: sobrepeso y obesidad se asociaron positivamente con la hipertensão arterial. El cálculo de la FAP permitió observar que, si se produjese una reducción o eliminación de hábitos y comportamientos relacionados con el estilo de vida de este público, se reduciría en un 56, $1 \%$ la prevalencia de la ECNT estudiada. La identificación de factores modificables y cómo pueden interferir negativamente en la salud de hombres trabajadores posibilita la planificación de intervenciones en el propio lugar de trabajo, con el fin de alcanzar al mayor número de individuos para reducir los efectos mortiferos de las ECNT.

Exámenes Médicos; Diagnóstico; Enfermedades No Transmisibles; Salud Laboral; Hombres
Recebido em 14/Out/2020

Versão final reapresentada em 27/Fev/2021

Aprovado em 15/Abr/2021 